XIV Simpósio Nacional de Sistemas Prediais

Gestão, Eficiência e Sustentabilidade

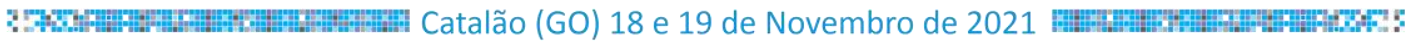

\title{
DIRETRIZES ARQUITETÔNICAS PARA O DESENVOLVIMENTO DE SISTEMAS DE APROVEITAMENTO DE ÁGUA PLUVIÁIS: UM MAPEAMENTO DE LITERATURA
}

\section{Architectural guidelines for the development of rainwater harvesting systems: a mapping study}

\author{
BARROS, Renata Lima'; CAMPOS, Marcus André Siqueira².
}

Recebido em 16 de julho de 2021, aprovado em 06 de setembro de 2021, publicado em 18 de novembro de 2021

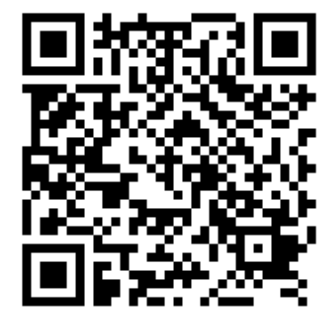

Palavras-chave:

Sistemas prediais de aproveitamento de águas pluviais.;

Arquitetura;

Diretrizes projetuais;

Revisão Sistemática.

Keywords:

Building systems for rainwater use;

Architecture;

Design Guidelines;

Systematic Review.

\begin{abstract}
RESUMO: A busca por construções verdes, que priorizem utilização de métodos voltados para preservação do meio ambiente, vem sendo uma prática cada vez mais comum no meio arquitetônico. No entanto ainda é pouco comum a utilização de sistemas prediais de aproveitamento de águas pluviais (SPAAP). Sua baixa popularidade deve-se, principalmente, ao seu elevado custo de implementação que, em diversos casos, acabam tornando-o inviável. No entanto, tais sistemas são de grande relevância, quando consideramos os atuais alertas de escassez de água do planeta para próximas décadas. É necessário o investimento em análises e pesquisas a respeito dos SPAAP, na busca por alternativas que possibilitem sua maior disseminação. Neste sentido, este trabalho apresenta uma Revisão Sistemática da Literatura acerca dos estudos já desenvolvidos, relacionando arquitetura e SPAAP.
\end{abstract}

\begin{abstract}
The search for green buildings, which prioritize the use of methods aimed at preserving the environment, is becoming an increasingly common practice in the architectural field. However, the use of rainwater harvesting systems (RWHS) is still uncommon. Its low popularity is mainly due to its high cost of implementation, which, in many cases, end up making it unfeasible. However, such systems are of great relevance when we consider the current warnings of water shortage on the planet for the next decades. It is necessary to invest in analysis and research about PHSS, in the search for alternatives that enable their wider dissemination. In this sense, this paper presents a Systematic Review of Literature about the studies already developed, relating architecture and RWHS.
\end{abstract}

\section{CONTATO DOS AUTORES:}

${ }^{1}$ BARROS, Renata Lima: Universidade Federal de Goiás, Brasil, renata_lima_barros@discente.ufg.br

${ }^{2}$ CAMPOS, Marcus André Siqueira: Universidade Federal de Goiás, Brasil, marcus_campos@ufg.br 


\section{INTRODUÇÃO}

Devido ao crescimento populacional, a crescente demanda por água, e as mudanças climáticas fazem com que os recursos hídricos diminuam a cada ano e começem a levar à escassez do recurso (MARIANA e SURYAWINATA, 2018). Segundo a ONU, em todo o mundo, cerca de três em cada dez pessoas, em um total de 2,1 bilhões, já não têm acesso a água potável. Além disto, a situação tende a agravar-se, uma vez que até 2030, devido ao crescimento populacional, se prevê um déficit de $40 \%$ deste recurso (ONU, 2017).

Portanto, uma abordagem sustentável aos recursos hídricos deve ser levada em consideração, pois a água é um recurso natural não renovável. Tudo isso, somados ainda ao alto consumo, o desperdício e a poluição da água. Neste contexto, destaca-se as vantagens da implementação do SPAAP, que possibilitam uma menor necessidade de fornecimento desse recurso por parte das companhias de saneamento. Tais sistemas ainda permitem uma economia das despesas de água e esgoto sendo sensível em todas as instâncias: residências unifamiliares, edifícios residenciais e comerciais, e principalmente indústrias, que geralmente possuem uma grande área de captação e diversos usos não potáveis.

Porém, de fato, a implementação de SPAAP demanda um alto investimento financeiro, elevando consideravelmente os custos de uma obra, o que, muitas vezes, pode inviabilizar o seu emprego. No entanto, seu custo é revertido ao longo dos anos, trazendo benefícios não só no aspecto financeiro como também ambientais e de conforto aos usuários (ALENCAR, 2004).

É necessário, é um maior investimento em análises e pesquisas a respeito dos SPAAP, na busca por alternativas maior disseminação. Considerando a importância desta discussão e pertinência do tema, este trabalho busca realizar um mapeamentode Literatura sobre o tema, auxiliando no desenvolvimento da tese proposta.

\section{METOdOLOGIA}

O mapeamento de Literatura é uma ferramenta de estudo que foca na busca por referencias bibliográficas que possam contribuir para o desenvolvimento de determinado tema. Para a evolução deste processo foi necessário buscar, selecionar e avaliar pesquisas que agregassem conhecimento, construindo o Estado da Arte do assunto.

Este artigo apresenta o caminho percorrido no estudo de mapeamento da Literatura sobre o tema: diretrizes projetuais para o desenvolvimento os Sistemas de Aproveitamento de Água. $O$ trabalho seguiu as seguintes etapas:

1. Planejamento - essa etapa baseia-se na melhor compreensão do tema. Como metodologia utilizou-se, primeiramente, a elaboração da questão de pesquisa e, então, a o preenchimento do protocolo de pesquisa por meio da estratégia PICOC;

2. Condução - essa é a etapa de seleção dos estudos aderentes;

3. Documentação - essa etapa de análise dos dados extraídos.

Após o trabalho de pesquisa e análise serão apresentados os resultados do trabalho no item 3 deste artigo. 


\subsection{Planejamento}

A etapa de planejamento baseia-se na identificação da necessidade e desenvolvimento de um protocolo de revisão. Para a identificação da necessidade foi necessária uma análise exploratória de conduzisse a compreensão do tema estudado.

A elaboração das questões de pesquisa foi a etapa seguinte. O foco principal da pesquisa é a compreensão de critérios arquitetônicos que arquitetônicos que influenciem no funcionamento dos sistemas de aproveitamento de águas pluviais. Sendo assim, elaborou-se as seguintes perguntas para a condução da pesquisa:

- Quais fatores influenciam na eficiência de um sistema de aproveitamento de águas pluviais?

- Quais questões arquitetônicas influenciam nos sistemas de aproveitamento de água pluvial?

\subsection{Condução}

Esta é a etapa de seleção dos estudos, baseados na elaboração da estratégia a ser utilizada na busca por pesquisas aderentes ao tema proposto. O primeiro passo foi a definição das palavras-chave que resumissem o assunto. Foram definas as palavras:

1) Sistemas prediais de aproveitamento de águas pluviais (Rainwater harvesting sistem);

2) Arquitetura (Architecture);

3) Diretrizes projetuais (project guidelines).

Após a escolhas das palavras, o próximo passo foi a elaboração do termo de busca (string) que melhor se adequasse ao tema. Após algumas simulações, a string escolhida foi Rainwater AND (harvest* OR collect) AND architecture AND (design OR project).

Assim, partiu-se para a próxima etapa: escolha das fontes de pesquisa. Foram selecionadas: Engineering Village, Science Direct, Scopus e Web of Science, que são bases de dados eletrônicas, mais comuns no meio acadêmico. Elas fornecem acesso a uma variedade de fontes com pesquisa acadêmicas, artigos científicos, periódicos, dentre outros.

A próxima etapa constituiu-se da seleção dos estudos. O processo foi feito com o auxílio do programa Parsifal. Trata-se de uma ferramenta online que auxilia na sistematização das referências. O programa organiza todos as literaturas encontradas nas bases, permitindo a visualização de seu título e autor. A sistematização feita pelo programa, nos permite separar manualmente as referências em: aceitas e rejeitadas. Neste trabalho os estudos foram selecionados da seguinte forma:

1. Primeiramente pela eliminação/seleção por leitura dos títulos - Através da leitura dos títulos das pesquisas encontradas, verificou-se previamente a aderência ou não da mesma ao tema estudado;

2. Após a eliminação por títulos, foi realizada eliminação por disponibilidade, pois alguns das literaturas selecionadas pelas bases de dados já não podem ser mais acessadas, 0 que acarretou na eliminação de tais literaturas;

3. A terceira etapa de seleção teve como critério a leitura dos resumos. Através da leitura de todos os resumos dos estudos selecionados na etapa anterior. Deste modo, foi possível uma análise mais acentuada. 


\subsection{Documentação}

A etapa de documentação consistiu na análise da qualidade e da relevância da literatura encontrada, compreendendo a pertinência de cada estudo à questão de pesquisa e ao foco da mesma. Para tanto, após a etapa de condução, partiu-se para a leitura dos estudos por completo, buscando a confirmação da aderência ou não ao tema estudado.

\section{RESULTADOS}

Seguindo a metodologia apresentada na seção anterior, foi realizada a busca da string nas bases de dados selecionada. Foram encontrados 58 publicações, sendo que delas:

1) 23 foram encontrados na base de dados Scopus;

2) 25 foram encontrados na base de dados ScienceDirect;

3) 10 foram encontrados na base de dados Web of Science.

Não foram encontradas literaturas na base Engineering Village. Também é importante ressaltar que não houve arquivos duplicados nas bases de dados. Realizando uma sistematização dos estudos disponibilizados em cada base o programa Parsifal gerou um o gráfico demonstrando a parcela de contribuição de cada base. Por meio do gráfico apresentado na Figura 1 percebe-se que a base Sicence Direct apresentou 43,1\% dos estudos ofertados, o Scopus $38,7 \%$ e o Web os Science $17,2 \%$, sendo somente essas as bases responsáveis pela oferta dos estudos aderentes.

Seguindo o método de avaliação apresentado na metodologia, do total de estudos encontrados, foram eliminados 41 arquivos por meio da leitura dos títulos, o que resultou em 17 publicações. Destas, 4 foram eliminadas por indisponibilidade de acesso e mais 8 por leitura de resumo. Deste modo, foram eliminados um total de 53 arquivos, restando apenas 5 para análise do conteúdo. A Figura 2 apresenta as etapas do processo de eliminações, assim como o descrito previamente na metodologia.

Figura 2 - Processo de seleção dos artigos.

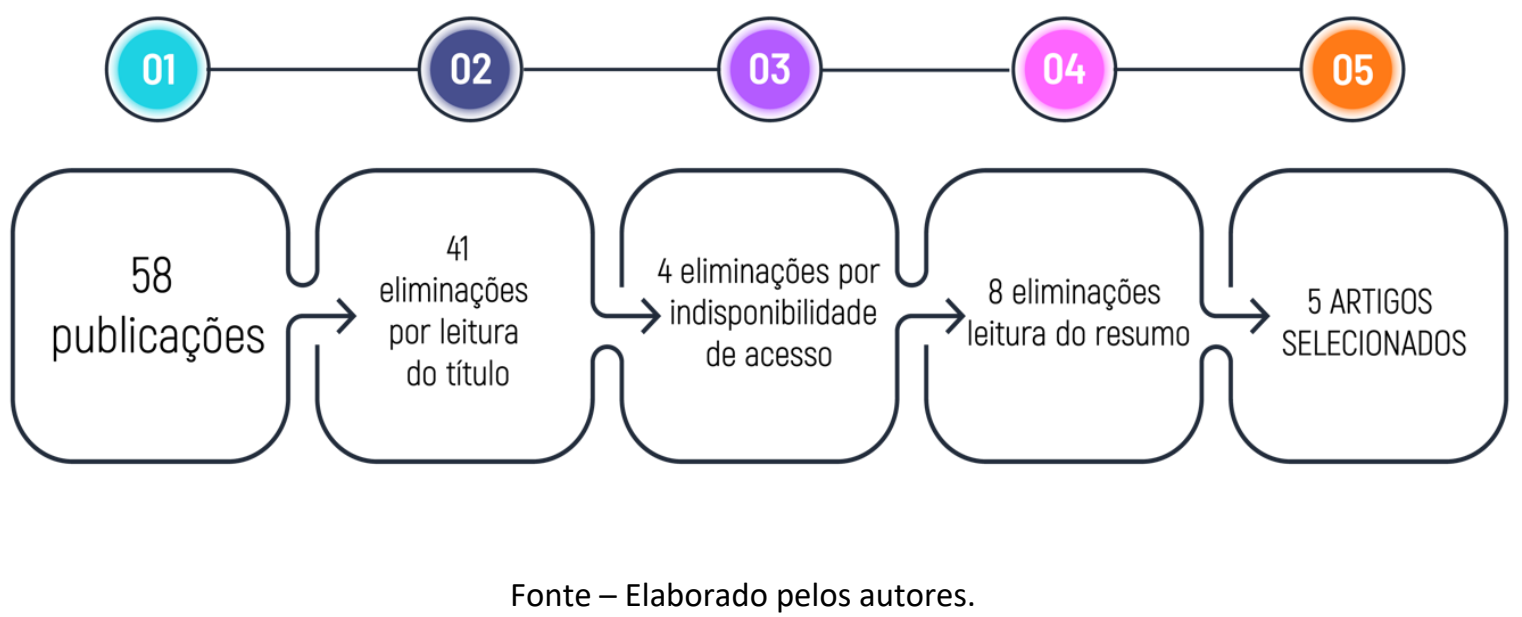

Quanto ao conteúdo propriamente dito, é possível perceber que todas as literaturas possuem o mesmo ponto de partida: a necessidade de desenvolvimento de sistemas de aproveitamento de águas pluviais como meio de solucionar a crise hídrica que já começa a surgir. Porém, apesar de todos tratarem a respeito de tal ponto chave, cada estudo trabalha com aspectos distintos sobre o assunto. 
Deste modo, a partir das literaturas selecionadas, foi possível construir um referencial teórico sobre pontos importantes em torno do tema principal, auxiliando de maneira significativa na construção da tese. A partir da análise de todas as literaturas foi possível selecionar seis principais pontos influenciadores no funcionamento dos SPAAP, sendo eles:

1) Questões climáticas - Se colocam como uma variante de grande importância quando o assunto é sistema de reaproveitamento de água. É uma questão que deve ser obrigatoriamente tratada, pois de acordo com cada clima temos um índice pluviométrico, e deste modo, uma quantidade de precipitações, o que influencia na quantidade de água coletada. Outra questão relacionada às questões climáticas é a quantidade de partículas de sujeira acumuladas. Em climas mais secos, há uma maior retenção, reduzindo a qualidade da água captada. Todas as pesquisas selecionadas citam as questões climáticas, porém os estudos dos autores Mariana \& Suryawinata (2018) e Shadmehri Toosi et al., (2020) o clima é um dos pontos chave das análises;

2) Demanda de água - Outro aspecto fundamental quando o tema está relacionado ao consumo de água. A ONU sugere uma recomendação mínima dos padrões de uso de água. A demanda é de essencial importância nos estudos de Mariana \& Suryawinata (2018); Shadmehri Toosi et al., (2020) e Şahin \& Manioğlu (2019);

3) Área de captação do telhado - Questão de grande importância quando o assunto é SAAP. $O$ aspecto é levado em consideração em todos os artigos aderentes. Porém no estudo realizado por Shadmehri Toosi et al., (2020) a área de captação é um dos principais eixos, demonstrando a importância nos estudos da área;

4) O material utilizado no telhado - O material em que é construído cada telhado influência tanto na qualidade da água captada (pois determinado materiais retém mais sujeira do que outros, e alguns liberam partículas indesejáveis). Este critério deve ser levado em consideração, e o estudo de Mao et al., (2021) comprova a influência deste critério;

5) Formas dos edifícios - No critério forma do edifício podemos englobar várias variantes como: inclinação dos telhados, inclinação das fachadas, forma do telado, dentre outras. Todas as bibliográficas elencadas citam ao menos algumas destas variantes como importantes para os estudos, principalmente inclinação do telhado. Porém, o estudo de Şahin \& Manioğlu (2019) foca nesta variante, realizando análises de acordo com a de acordo com diferentes formas;

6) Práticas comuns dos SAAP - Tema eixo do estudo realizado por Thomas et al., (2014). Trata-se da identificação dos padrões mais utilizados em Sistemas de Aproveitamento de Águas Pluviais nos Estados Unidos, como os materiais mais utilizados, o tipo de sistema mais utilizado.

Deste modo, a partir das literaturas selecionadas, foi possível construir um referencial teórico sobre pontos importantes em torno do tema principal, auxiliando de maneira significativa na construção da tese.

Os esquemas da Figura 3 apresentam visualmente os seis eixos de estudo e relaciona a referência que construiu um estudo a respeito de cada tema. No esquema 1 , temos os seis principais temas relacionados presentes em estudos a respeito SPAAP, e nos esquemas 2, 3, 4, 5 e 6 são apresentados com a linha pontilhada quais os temas-eixos de cada artigo, - escritos na cor da correspondente linha pontilhada. Assim como o dito, todos os artigos citam a importância de todos os critérios, mas cada um foca seu estudo em um ou dois deles. O estudo de Mariana e Suryawanta (2018), por exemplo, engloba um pouco de cada um dos temas, mas foca nas questões climáticas e demanda de água. 
Figura 3 - Conexão dos eixos temáticos com os estudos aderentes.

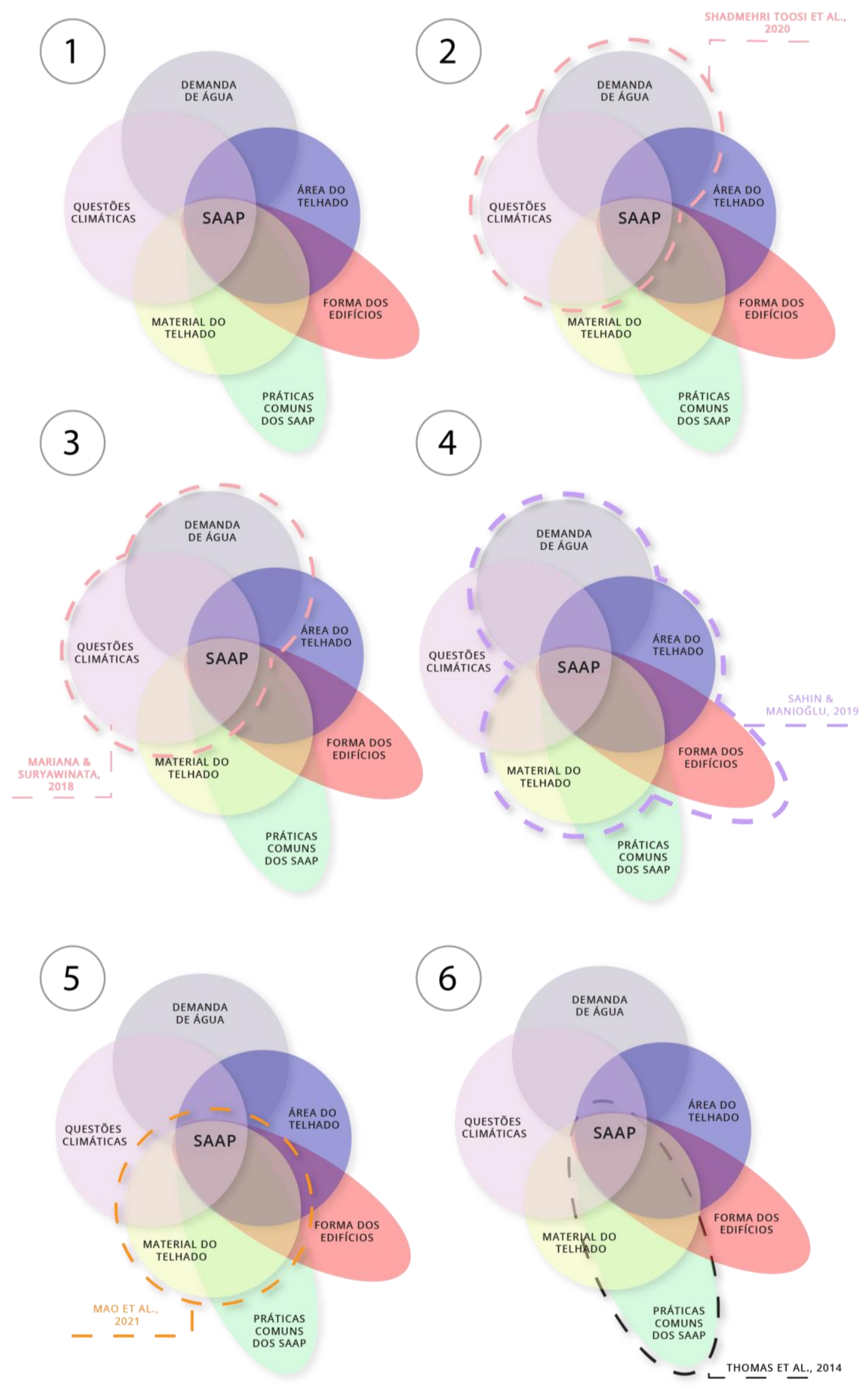

Fonte - Elaborado pelos autores. 


\section{CONSIDERAÇÕES FINAIS}

Por meio do mapeamento da Literatura realizado foi possível construir uma fundamentação teórica a respeito das influências arquitetônicas na implementação dos Sistemas de Aproveitamento de Águas Pluviais. Foi possível identificar, selecionar e avaliar criticamente estudos que se relacionam com o tema, auxiliando na construção dos próximos passos para o desenvolvimento de tese.

A sistematização das literaturas permitiu a constatação de que não há a ocorrência massiva de estudos a respeito do tema, sendo encontrados somente 5 estudos aderentes, em 4 bases eletrônicas de pesquisas mais utilizadas no meio acadêmico. Deste modo, foi possível perceber a necessidade da utilização de outras strings mais amplas para complementação da revisão bibliográfica da tese. É necessário o desenvolvimento de pesquisas que tragam uma maior quantidade de literatura para a construção da tese.

\section{REFERÊNCIAS}

ASSOCIAÇÃO BRASILEIRA DE NORMAS TÉCNICAS. NBR15575: Edificações habitacionais - Desempenho. Rio de Janeiro, 2013.

ABSOLAR, Associação Brasileira de Energia Solar Fotovoltaica. Disponível em: http://www.absolar.org.br / Acesso em: 25 de maio de 2020.

ALENCAR, C. T.; O equilíbrio entre a sustentabilidade e a atratividade do investimento em edifícios de escritórios para locação em São Paulo; ENTAC, São Paulo Brasil; 2004.

CAMPOS, M. A. S. Aproveitamento de água pluvial em edifícios residenciais multifamiliares na cidade de São Carlos. 2004, 131p. Dissertação (Mestrado em Engenharia Civil) - Universidade Federal de São Carlos, São Carlos, 2004.

CAMPOS, M. A. S. Qualidade de investimentos em sistemas prediais de aproveitamento de água pluvial: uso de partículas swarm optimization. 2012, 95p. Tese (Doutorado em Engenharia Civil) Faculdade de Engenharia Civil, Arquitetura e Urbanismo, Universidade Estadual de Campinas. Campinas, 2012.

CAMPOS, M. A. S.; AMORIM, S. V. In: IV SIMPÓSIO BRASILEIRO DE GESTÃO E ECONOMIA DA CONSTRUÇÃO, 2005, Florianópolis. Anais... 2005.

CAMPOS, M. A. S.; ILHA, M. S. O. Dimensionamento de reservatórios para o aproveitamento de água pluvial: critérios econômicos. In: XIII ENCONTRO NACIONAL DE TECNOLOGIA DO AMBIENTE CONSTRUÍDO, 2010, Canela, RS. Anais... 2010.

CAMPOS, M. A. S.; ILHA, M. S. O.; GRANJA, A. D. Investimento em sistemas de aproveitamento de água pluvial: Estudo de caso para uma edificação residencial multifamiliar no município. In: V SIMPÓSIO BRASILEIRO DE GESTÃO E ECONOMIA DA CONSTRUÇÃO, 2007, Campinas, SP. Anais... Campinas, 2007.

COLLA, L.L., Sistemas de Captação e Aproveitamento de Água de Chuva . 2003. Trabalho de Conclusão de Curso (Graduação de Engenharia Ambiental) - Faculdade de Engenharia Civil, UNESP. Sorocaba, 2008.

COSTA, S.C., SCOCUGLIA, J.B.C., Diretrizes de sustentabilidade na arquitetura. Disponível em www.vitruvius.com.br. Acesso em 13 mar.2014.

CUNHA, Eduardo Grala da, Discussão sobre o papel da tecnologia no processo de concepção arquitetônica contemporânea: o caso Norman Foster. Disponível em www.vitruvius.com.br. Acesso em 13 mar.2014. 
DEMANTOVA, Graziella, Sustentabilidade e o futuro das cidades. Disponível em www.vitruvius.com.br. Acesso em 13 mar.2014.

DRESCH, Aline. LACERDA, Daniel Pacheco. ANTUNES, José Antônio Valle Júnior. Design Science Research: Método de pesquisa para avanço da ciência e tecnologia. Porto Alegre: Bookman, 2015. $173 p$.

FLACH, Fernanda. GONZÁLEZ, Marco Aurélio Stumpf. KERN, Andrea Parisi. A knowledge discovery mechanism to user requirement identification in building design. Revista Ingeniería de Construcción, $v$ 27, n 2, p. 83-98, 2012.

INCITIES JOURNAL CITATION REPORTS. InCities Journal Citation Reports. Disponível em: <https://jcrclarivate.ez49.periodicos.capes.gov.br/JCRLandingPageAction.action?lnit=Yes\&SrcApp=IC2

LS\&SID=H4-slyvnoJ1ixxx2FqwdV4U6tQDwFx2BlePwdjJH-

18x2dLph5jGqnrwCix2BC4bxxs5fRAx3Dx3Dvk81sp7FAuloQXbTwGlu4Ax3Dx3D-

qBgNuLRjcgZrPm66fhjx2Fmwx3Dx3D-h9tQNJ9Nv4eh45yLvkdX3gx3Dx3D> Acesso em: 22 dez. 2019.

LAMBERTS, R. Eficiência Energética na Arquitetura, Sao Paulo : Pro Livros, 2004.

MACOMBER, P. S. H. Guidelines on rainwater catchment systems for Hawaii. 2010. 52 p. ISBN: 1929325-23-1.

MAO, J., XIA, B., ZHOU, Y., BI, F., ZHANG, X., ZHANG, W., \& XIA, S. (2021). Effect of roof materials and weather patterns on the quality of harvested rainwater in Shanghai, China. Journal of Cleaner Production, 279, 123419. https://doi.org/https://doi.org/10.1016/j.jclepro.2020.123419

MARIANA, Y., \& SURYAWINATA, B. A. (2018). Sustainable water management: Roof as a rainwater catchment area. IOP Conference Series: Earth and Environmental Science, 195(1). https://doi.org/10.1088/1755-1315/195/1/012094

ŞAHIN, N. I.., \& MANIOĞLU, G. (2019). Water conservation through rainwater harvesting using different building forms in different climatic regions. Sustainable Cities and Society, 44, 367-377. https://doi.org/https://doi.org/10.1016/j.scs.2018.10.010

RAMOS, R.; Sustentabilidade e economia. Revista Casa e Construção, Rio de Janeiro, n. 55, 2010.

RAPOPORT, A. Origens culturais da arquitetura. In: SNYDER, J. C.; CATANESE, A. Introdução à arquitetura. Rio de Janeiro, Editora Campus, P 26 41, 1984.

RATTNER, H.; Meio ambiente, saúde e desenvolvimento sustentável. Ciência e saúde coletiva, São Paulo, v 14, n.6, p.1965 1971, 2009.

ROCHA, B. C. C. M; Avaliação do desempenho do sistema de descarte de água de chuva em coberturas de três diferentes tipos de materiais. 2010. 186f. Dissertação (Mestrado em Engenharia do Meio Ambiente) Programa de Pós-Graduação em Engenharia do Meio Ambiente da Escola de Engenharia Civil da Universidade Federal de Goiás. Goiânia, 2010.

SHADMEHRI TOOSI, A., GHASEMI TOUSI, E., GHASSEMI, S. A., CHESHOMI, A., \& ALAGHMAND, S. (2020). A multi-criteria decision analysis approach towards efficient rainwater harvesting. Journal of Hydrology, 582, 124501. https://doi.org/https://doi.org/10.1016/j.jhydrol.2019.124501

SILVA, L. M. Estudo da demanda de água não potável e desenvolvimento de um sistema de reuso de águas cinzas para habitações de interesse social. 2013. 157 f. Dissertação (Mestrado em Engenharia Ambiental) Universidade Federal do Espírito Santo, Vitória, 2013.

SNIS, Sistema Nacional de Informações sobre Saneamento. Disponível em: < www.snis.gov.br/ >. Acessado em 3 de novembro de 2016. 
TEXAS WATER DEVELOPMENT BOARD; Texas Manual On Rainwater Harvesting; Third Edition, Austin Texas EUA. 2005. 88 p.

THOMAS, R. B., KIRISITS, M. J., LYE, D. J., \& KINNEY, K. A. (2014). Rainwater harvesting in the United States: a survey of common system practices. Journal of Cleaner Production, 75, 166-173. https://doi.org/https://doi.org/10.1016/j.jclepro.2014.03.073

VAN LENGEN, J. Manual do arquiteto descalço. São Paulo: Empório do Livro, 2008.

ONU, Organização das Nações Unidas. Disponível em: < www.nacoesunidas.org >. Acessado em 22 de maio de 2020. 